\title{
Poluição do ar e impactos na saúde na Região Metropolitana de Belo Horizonte - Minas Gerais, Brasil
}

\author{
Air pollution and the impacts on health in the Metropolitan Region \\ of Belo Horizonte in the State of Minas Gerais, Brazil
}

Nelson Gouveia (http://orcid.org/0000-0003-0625-0265) ${ }^{1}$

Antonio Ponce de Leon (https://orcid.org/0000-0002-0704-5215) ${ }^{2}$

Washington Junger (https://orcid.org/0000-0002-6394-6587) ${ }^{2}$

Joana de Freitas Lins (http://orcid.org/0000-0003-1209-1440) ${ }^{3}$

Clarice Umbelino de Freitas (http://orcid.org/0000-0002-6742-3409) ${ }^{4}$

\footnotetext{
${ }^{1}$ Departamento de Medicina Preventiva, Universidade de São Paulo. Av. Dr. Arnaldo, 455, 20 andar. 01246-903 São Paulo SP Brasil. ngouveia@usp.br

${ }^{2}$ Instituto de Medicina Social, Universidade do Estado do Rio de Janeiro. Rio de janeiro RJ Brasil. ${ }^{3}$ Faculdade Pernambucana de Saúde. Recife PE Brasil. ${ }^{4}$ Laboratório de Investigação Médica, Hospital das Clínicas da FMUSP. São Paulo SP Brasil.
}

\begin{abstract}
The impact of air pollution on hospitalizations for total respiratory diseases (DRT) among children under five (DRC), as well as for cardiovascular diseases (CVD) in patients over 39 years of age, was examined in the municipalities of Belo Horizonte, Betim and Contagem, of the Metropolitan Region of Belo Horizonte, Minas Gerais. Generalized additive models using Poisson regression were used to adjust the daily time series of hospitalizations. Single and accumulated lagged pollutants of up to five days were introduced as independent variables and models were adjusted for temperature, humidity, weekdays and holidays. In Belo Horizonte PM10 was related to DRT (RR\% 1.06 CI 95\%: 0.41-1.72); DRC (\%RR 1.25 CI 95\%: 0.25-2.26) and CVD (RR\% 2.29, CI 95\%: 0.96-3.64). In Betim an RR\% of 1.33 (CI 95\%: 0.48-2.18) for DRT and RR\% 2.38 (CI 95\%: 1.20-3.56) for DRC was observed. In Contagem $R R \%=1.23$ (CI 95\%: 0.32-2.15) and $R R \%=1.61$ (CI 95\%: 0.26-2.96) was observed for DRT and DRC, respectively. SO2 and CO were also associated with hospitalizations. Respiratory diseases were the outcome most frequently related to air pollutants investigated. These results can be useful in discussions on emission control policies in the region.
\end{abstract}

Key words Air pollution, Respiratory diseases, Cardiovascular diseases, Time series
Resumo Examinamos o impacto da poluição atmosférica nas internações por doenças respiratórias totais (DRT), em crianças menores de cinco anos (DRC) e por doenças cardiovasculares em maiores de 39 anos (DCV) nos municípios de Belo Horizonte (BH), Betim e Contagem, da Região Metropolitana de Belo Horizonte. Modelos aditivos generalizados via regressão de Poisson foram utilizados para ajustar as séries de hospitalizações. Os poluentes, em defasagem simples e acumulada de até cinco dias, foram introduzidos como variáveis independentes e os modelos foram ajustados para temperatura, umidade, dias da semana e feriados. Em BH, o $\mathrm{PM}_{10}$ esteve relacionado às DRT (RR\% 1,06 IC95\%:0,41-1,72); DRC (RR\% 1,25 IC95\%:0,25-2,26) e DCV (RR\% 2,29 IC95\%:0,96-3,64). Em Betim, observou-se RR\% 1,33 (IC95\%:0,48-2,18) para DRT e RR\% 2,38 (IC95\%:1,20-3,56) para DRC. Em Contagem, observou-se RR\% 1,23 (IC95\%:0,32-2,15) e RR\% 1,61 (IC95\%:0,26-2,96) para DRT e DRC, respectivamente. $\mathrm{SO}_{2}$ e CO também apresentaram associação com as hospitalizações. As doenças respiratórias foram o desfecho mais relacionado aos poluentes investigados. Esses resultados podem ser úteis nas discussões das politicas de controle de emissões na região.

Palavras-chave Poluição do ar, Doenças respiratórias, Doenças cardiovasculares, Séries temporais 


\section{Introdução}

Os impactos da poluição do ar na saúde têm sido tema de estudo em diversas localidades no mundo, particularmente seus efeitos sobre o sistema cardiovascular e no aparelho respiratório, para os quais ainda não se estabeleceu limite seguro ${ }^{1}$. O sistema respiratório é a principal porta de entrada de poluentes do ar e, consequentemente, seus efeitos vêm sendo relatados há décadas. Evidências epidemiológicas consistentes têm indicado que exposições a curto e longo prazos a poluentes atmosféricos como o material particulado (PM) ozono (O3) e óxidos de nitrogênio (NOx) estão associadas a aumentos na mortalidade respiratória ${ }^{2,3}$. Também há evidências de que a exposição a poluentes atmosféricos está associada a doenças respiratórias crônicas como a $\mathrm{DPOC}^{4}$. De modo similar, numerosos estudos vêm indicando associações entre exposições a poluentes atmosféricos e eventos cardiovasculares agudos, bem como biomarcadores de processos cardiovasculares patogênicos 5 .

Dentre os tipos de estudos que avaliam os efeitos da poluição do ar, os estudos ecológicos de séries temporais têm sido os mais utilizados. No Brasil, particularmente nas cidades de São Paulo e Rio de Janeiro os estudos de séries temporais têm contribuído para o debate sobre os efeitos deletérios da poluição do $\operatorname{ar}^{6}$.

Como uma das regiões brasileiras mais ricas em recursos minerais, a Região Metropolitana de Belo Horizonte (RMBH) é composta por 33 municípios e responsável por $66 \%$ da atividade mineradora do estado de Minas Gerais, destacando-se a extração de minério de ferro, manganês, ouro e calcário. As indústrias predominantes são do setor siderúrgico, de minerais não metálicos (cimento e cal), de petróleo e a indústria automobilística. ${ }^{7}$ A população da $\mathrm{RMBH}$ representa $40 \%$ da população de Minas Gerais e responde por cerca de $34 \%$ do Produto Interno Bruto (PIB) do estado. Com população estimada pelo Instituto Brasileiro de Geografia e Estatística (IBGE), para 2015, em 2,5 milhões de habitantes, 113 mil empresas atuantes e frota veicular, em 2014, de 1,6 milhões numa área de $331 \mathrm{mil} \mathrm{km}^{2}$, Belo Horizonte apresenta característica mista de poluição ${ }^{8}$.

Os municípios de Betim e Contagem possuem relação veículo/população menor que a capital $(0,15 \text { e } 0,22 \text { respectivamente })^{8}$, o que faz supor que a poluição atmosférica proveniente das indústrias tenha peso maior. Por outro lado, a localização das estações de monitoramento da qualidade do ar e sua área de abrangência influenciam nos níveis dos parâmetros aferidos e podem não refletir a complexidade da dispersão dos poluentes atmosféricos. De acordo com a Fundação Estadual de Meio Ambiente (FEAM) de Minas Gerais, as estações de monitoramento de Belo Horizonte estão localizadas em áreas de intenso fluxo de veículos, tendo maior influência desta modalidade poluidora. Em Betim, que conta com três estações de monitoramento da qualidade do ar, duas das estações localizam-se em área residencial, e a outra próxima a uma refinaria. A estação de monitoramento da qualidade do ar localizada no Departamento Nacional de Estradas e Rodagem (DNER), em Contagem, é circundada por diversas indústrias além de concentrar tráfego intenso de veículos pesados ${ }^{7}$.

Até o momento não foram encontrados estudos de relação entre poluição do ar e efeitos na saúde na $\mathrm{RMBH}$. Buscou-se no presente trabalho, avaliar o impacto da poluição do ar em três municípios da RMBH que contam com monitoramento da qualidade do ar (Figura 1) Belo Horizonte, Betim e Contagem, utilizando a mesma metodologia empregada em outras cidades brasileiras, o que permitirá comparações futuras.

\section{Metodologia}

Este estudo faz parte do Projeto de Avaliação de Impacto da Poluição do Ar nas Cidades Brasileiras, que visou examinar o impacto da poluição do ar na saúde com o objetivo de propor ações de vigilância epidemiológica. Em todas as cidades que aderiram ao projeto, dados de internações hospitalares, conforme as Autorizações de Internação Hospitalar (AIH), segundo local de residência de 2001 a 2006, foram obtidos do Sistema de Informações Hospitalares (SIH) do Departamento de Informática do SUS (DATASUS). Estes dados foram inseridos em um portal de informações construído no âmbito do projeto com a finalidade de facilitar a seleção de períodos de estudo com maior completude de dados dos parâmetros ambientais. Essas informações foram agrupadas por dia, ou seja, contagens diárias de internações pelas causas investigadas: doenças respiratórias (CID10: J00-J99) em todas as idades (DRT) e em menores de 5 anos (DRC) e doenças cardiovasculares (CID10: I00-I99) em maiores de 39 anos (DCV).

No mesmo portal foram agregadas as concentrações de poluentes segundo estações de monitoramento e os parâmetros meteorológicos. 

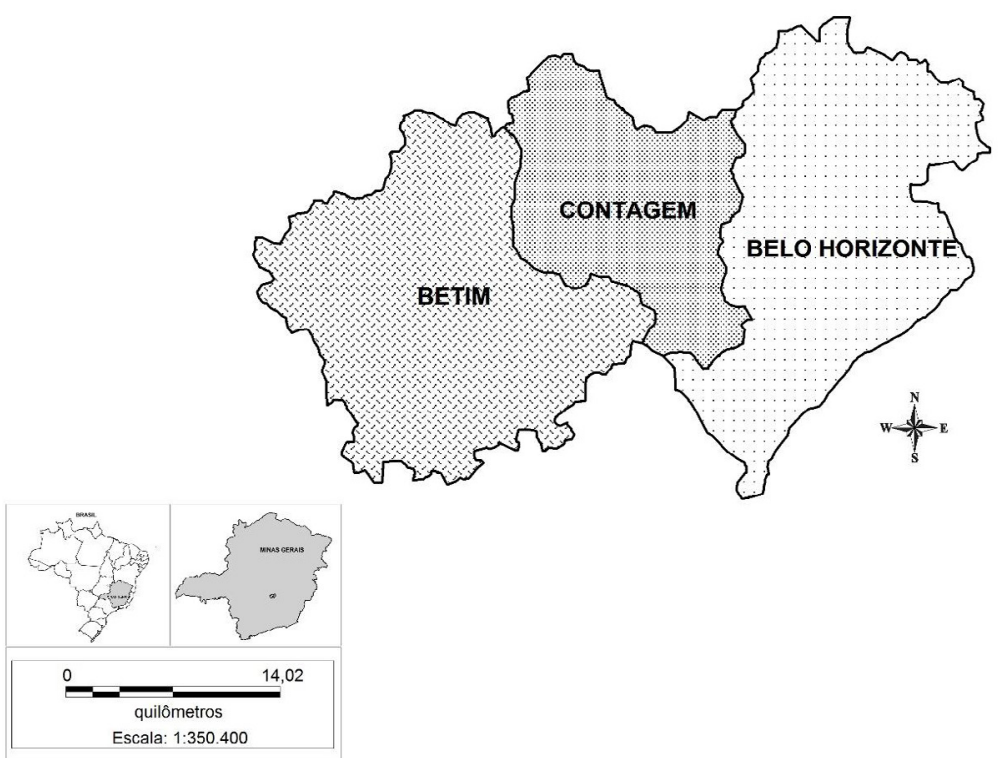

Figura 1. Municípios estudados na Região Metropolitana de Belo Horizonte, Minas Gerais.

Como variável de exposição se utilizou as concentrações médias diárias de material particulado com até $10 \mu \mathrm{m}$ de diâmetro $\left(\mathrm{PM}_{10}\right)$, monóxido de carbono (CO) e dióxido de enxofre $\left(\mathrm{SO}_{2}\right)$ e, como controles, foram introduzidas nos modelos a temperatura e a umidade relativa do ar.

Nos municípios de Belo Horizonte, Betim e Contagem os dados meteorológicos (temperatura e umidade relativa do ar) e os dados de poluentes $\left(\mathrm{PM}_{10}, \mathrm{CO}\right.$ e $\left.\mathrm{SO}_{2}\right)$ foram cedidos pela $\mathrm{FEAM}^{7}$. As bases foram avaliadas quanto à continuidade das informações, aceitando-se perdas de no máximo $15 \%$ dos dias no período para cada variável ambiental. $\mathrm{O}$ período contínuo com menor proporção de falhas dentro do critério estabelecido foi selecionado para análise. Apesar de contar com estação de monitoramento da qualidade do ar, a cidade de Ibirité, pertencente a RMBH, não foi incluída no estudo devido à grande descontinuidade dos dados. Os dados das estações de monitoramento de Belo Horizonte apresentavam melhor continuidade de informações entre julho de 2004 a dezembro de 2006 tendo sido analisados o $\mathrm{PM}_{10}$, $\mathrm{CO}$ e $\mathrm{SO}_{2}$. Em Betim foi feita análise no período de janeiro de 2002 a dezembro de 2006, tendo apenas o $\mathrm{PM}_{10}$ preenchido os critérios de completude. Em Contagem, o período de dezembro de 2004 a novembro de 2006 foi o que apresentou menor proporção de falhas de informação para o $\mathrm{PM}_{10} \mathrm{eSO}_{2}$.
Foi realizada análise de séries temporais com a biblioteca ares desenvolvida para o aplicativo $\mathrm{R}^{9}$, utilizando metodologia comum para as cidades que aderiram ao projeto.

Foram construídos modelos explicativos para contagens de internações pelas causas estudadas ao longo do tempo. Os modelos propostos pertencem à classe de Modelos Aditivos Generalizados (GAM), com a opção de regressão de Poisson, segundo a equação:

$$
\ln \left(E\left(Y_{t}\right)=\beta X_{1 t}+\sum_{i=2}^{p} S_{i}\left(X_{i t}\right)\right.
$$

onde $Y_{t}$ e $X_{1 t}$ são os números de eventos e o nível de um dado poluente no dia $\mathrm{t}$, respectivamente. No estudo, $2 \leq i \leq p,\left\{X_{i t}\right\}$ são as variáveis temporais preditoras da resposta $Y_{t}$, uma das quais é a variável indicadora do tempo decorrido desde o início do período; e $2 \leq i \leq p,\left\{S_{i}\right\}$ são as funções de suavização. Aqui, as funções de suavização usadas foram as splines naturais. Foram acrescentadas variáveis indicadoras para os dias da semana e feriados nacionais ou locais. Os feriados com significância de até 0,09 foram agrupados segundo a direção do seu efeito: positiva ou negativa. No processo de modelagem da série temporal buscou-se minimizar o AIC (Critério de Informação de Akaike) e otimizar a Função de Auto Correlação Parcial (PACF). 
A sazonalidade e variáveis meteorológicas foram ajustadas por splines naturais, usando o número de graus de liberdade necessários para otimizar o AIC e o PACF. O propósito das funções de suavização é remover padrões sazonais e ajustar o efeito das variáveis meteorológicas.

Após a construção do modelo de trabalho (baseline) contendo todas as variáveis de controle e da verificação de sua adequação, foram introduzidos, individualmente, os poluentes em defasagens simples de até cinco dias e verificado também o efeito cumulativo neste período, utilizando um modelo polinomial de defasagens distribuídas. Este modelo, além de considerar a latência do efeito dos poluentes, minimiza a instabilidade no processo de estimação, próprio das análises que utilizam múltiplas defasagens ${ }^{10}$.

$\mathrm{O}$ efeito foi estimado para cada poluente introduzido ao modelo de trabalho de forma linear, obtendo-se um coeficiente ( $\beta$ ) de cada modelo de regressão, que por exponenciação fornece o risco relativo (RR). Por fim, obteve-se o risco relativo percentual (RR\%) para cada incremento de $10 \mu \mathrm{g} / \mathrm{m}^{3}$, o qual é derivado a partir da fórmula: $\mathrm{RR} \%=\left(e^{10 \beta}-1\right)^{\star} 100$. Esta quantidade expressa a variação percentual no número médio de internações hospitalares para uma variação de 10 $\mu \mathrm{g} / \mathrm{m}^{3}$ na concentração do poluente. Em todos os testes de hipóteses foi assumido um nível de significância de 5\%.

Este projeto foi aprovado pelo Comitê de Ética em Pesquisa do Hospital das Clínicas da Faculdade de Medicina da Universidade de São Paulo.

\section{Resultados}

A Tabela 1 apresenta as análises descritivas básicas das cidades estudadas, onde pode-se observar que a cidade de Betim, apesar de contar com maior período de análise, também conta com maior número de dias com ausência de dados de $\mathrm{PM}_{10}(13 \%)$, único poluente avaliado. Em Contagem, as perdas de dias para análise do $\mathrm{PM}_{10}$ chegaram a $11 \%$ e em Belo Horizonte as perdas foram mínimas $(0,2 \%)$. Para o $\mathrm{SO}_{2}$ e CO as perdas de dias para análise ficaram em torno de $3 \%$. Os níveis médios de $\mathrm{PM}_{10}$ e $\mathrm{SO}_{2}$ foram mais elevados em Contagem, tendo a cidade de Betim ocupado posição intermediária quanto aos níveis de material particulado inalável.

A cidade de Belo Horizonte contou com um elevado número médio diário de internações por todas as causas pesquisadas. Por serem cidades de menor população, Betim e Contagem apresenta- ram valores médios diários de internação menores, com diversos dias sem internação, particularmente das doenças respiratórias em menores de 5 anos, o que dificultou o ajuste dos modelos. Em Betim, onde houve maior dificuldade de ajuste, foi necessário a introdução de uma variável indicadora do ano de estudo para melhor controle da PACF.

As Tabelas 2, 3 e 4 apresentam os efeitos da poluição nas internações por doenças respiratórias e cardiovasculares, respectivamente para Belo Horizonte, Betim e Contagem. Poucos RR\% das análises utilizando defasagens individuais foram estatisticamente significantes (dados não apresentados). Particularmente para as doenças respiratórias totais, essas análises mostram um padrão de aumento no risco até a defasagem de 2 dias, seguido de um decréscimo desse risco. Resultados mais consistentes e de maior magnitude foram observados nas análises com modelo polinomial de defasagens distribuídas, no acumulado de 5 dias. Para um aumento de $10 \mu \mathrm{g} / \mathrm{m}^{3}$ nos níveis de $\mathrm{PM}_{10}$ observa-se um aumento de $6 \%$, $33 \%$ e $23 \%$ nas internações por doenças respiratórias totais nas cidades de Belo Horizonte, Betim e Contagem, respectivamente.

Em Belo Horizonte, onde há dados sobre 3 diferentes poluentes atmosféricos, os maiores riscos foram observados para o CO, seguido do $\mathrm{SO}_{2}$ e $\mathrm{PM}_{10}$ para os 3 desfechos estudados. Para um aumento de $1 \mathrm{ppm}$ nos níveis de $\mathrm{CO}$ e de $10 \mu \mathrm{g} /$ $\mathrm{m}^{3}$ nos níveis de $\mathrm{SO}_{2}$ e $\mathrm{PM}_{10}$ observamos um aumento percentual de 8 vezes, 2,6 vezes e de $6 \%$ nas internações por doenças respiratórias totais.

Embora com RR\% menores que os demais poluentes, o $\mathrm{PM}_{10}$ esteve associado a incrementos de internações por doenças respiratórias também em menores de cinco anos nas três cidades estudadas.

Com relação às internações por doenças cardiovasculares em maiores de 39 anos, observamos incrementos relacionados ao $\mathrm{PM}_{10}$ e $\mathrm{SO}_{2}$ apenas em Belo Horizonte, com aumentos de 2,3 e 5,9 vezes nas internações por essas doenças relativos a aumentos de $10 \mu \mathrm{g} / \mathrm{m}^{3}$ nos níveis de $\mathrm{PM}_{10}$ e $\mathrm{SO}_{2}$.

\section{Discussão}

O presente estudo é o primeiro a abordar o impacto da poluição do ar na saúde na $\mathrm{RMBH}$, onde foram avaliadas as internações por doenças respiratórias e cardiovasculares em três municípios que contam com monitoramento diário da 
Tabela 1. Parâmetros descritivos básicos - Belo Horizonte, Betim e Contagem - Minas Gerais, Brasil.

\begin{tabular}{|c|c|c|c|c|c|c|c|c|c|}
\hline Parâmetro & $\begin{array}{l}\text { Número de } \\
\text { dias com } \\
\text { dados }\end{array}$ & na $^{a}$ & Média & $\mathbf{D P}^{\mathrm{b}}$ & $\min$ & $\max$ & $\mathrm{p} 25^{\mathrm{c}}$ & ${\mathrm{p} 50^{\mathrm{d}}}^{\mathrm{d}}$ & $\mathrm{p} 75^{\mathrm{e}}$ \\
\hline \multicolumn{10}{|c|}{ Belo Horizonte: julho de 2004 a dezembro de 2006} \\
\hline Doenças respiratórias totais & 914 & 0 & 41,9 & 15,5 & 8 & 90 & 31 & 41 & 51 \\
\hline $\begin{array}{l}\text { Doenças respiratórias em } \\
\text { menores de } 5 \text { anos }\end{array}$ & 914 & 0 & 18,5 & 10,5 & 2 & 58 & 11 & 16 & 23 \\
\hline $\begin{array}{l}\text { Doenças cardiovasculares em } \\
\text { maiores de } 39 \text { anos }\end{array}$ & 914 & 0 & 35,8 & 14,1 & 10 & 113 & 27 & 35 & 42 \\
\hline $\operatorname{PM} 10\left(\mu \mathrm{g} / \mathrm{m}^{3}\right)$ & 912 & 2 & 19,6 & 10,8 & 2,5 & 80,2 & 12,4 & 17,4 & 24,6 \\
\hline $\mathrm{CO}(\mathrm{ppm})$ & 879 & 35 & 0,5 & 0,2 & 0,1 & 2,9 & 0,3 & 0,4 & 0,6 \\
\hline $\mathrm{SO} 2\left(\mu \mathrm{g} / \mathrm{m}^{3}\right)$ & 884 & 30 & 0,5 & 0,7 & 0 & 4,9 & 0 & 0,2 & 0,7 \\
\hline $\mathrm{T}\left({ }^{0} \mathrm{C}\right)$ & 909 & 5 & 21,9 & 2,6 & 14,6 & 29,6 & 20,1 & 22,1 & 23,9 \\
\hline UR $(\%)$ & 909 & 5 & 73,1 & 11,1 & 38,8 & 98,5 & 65,6 & 73,1 & 80,6 \\
\hline \multicolumn{10}{|c|}{ Betim: janeiro de 2002 a dezembro de 2006} \\
\hline Doenças respiratórias totais & 1826 & 0 & 6,4 & 3,6 & 0 & 25 & 4 & 6 & 8 \\
\hline $\begin{array}{l}\text { Doenças respiratórias em } \\
\text { menores de } 5 \text { anos }\end{array}$ & 1826 & 0 & 3,4 & 2,7 & 0 & 17 & 2 & 3 & 5 \\
\hline $\begin{array}{l}\text { Doenças cardiovasculares em } \\
\text { maiores de } 39 \text { anos }\end{array}$ & 1826 & 0 & 3,6 & 2,1 & 0 & 12 & 2 & 3 & 5 \\
\hline $\operatorname{PM} 10\left(\mu \mathrm{g} / \mathrm{m}^{3}\right)$ & 1585 & 241 & 35,7 & 20,4 & 3,0 & 139,7 & 21,1 & 30,1 & 45,2 \\
\hline $\mathrm{T}\left({ }^{0} \mathrm{C}\right)$ & 1554 & 272 & 22,4 & 3,0 & 13,6 & 30,9 & 20,5 & 22,4 & 24,4 \\
\hline UR (\%) & 1554 & 272 & 72,5 & 12,5 & 31,6 & 99,3 & 64,1 & 72,2 & 80,9 \\
\hline \multicolumn{10}{|c|}{ Contagem: dezembro de 2004 a novembro de 2006} \\
\hline Doenças respiratórias totais & 730 & 0 & 8,3 & 4,3 & 0 & 27 & 5 & 8 & 11 \\
\hline $\begin{array}{l}\text { Doenças respiratórias em } \\
\text { menores de } 5 \text { anos }\end{array}$ & 730 & 0 & 4,1 & 3,1 & 0 & 20 & 2 & 3 & 6 \\
\hline $\begin{array}{l}\text { Doenças cardiovasculares em } \\
\text { maiores de } 39 \text { anos }\end{array}$ & 730 & 0 & 6,3 & 2,9 & 0 & 18 & 4 & 6 & 8 \\
\hline $\operatorname{PM} 10\left(\mu \mathrm{g} / \mathrm{m}^{3}\right)$ & 649 & 81 & 44,1 & 20,8 & 8 & 163 & 30,1 & 39,8 & 52,1 \\
\hline $\mathrm{SO} 2\left(\mu \mathrm{g} / \mathrm{m}^{3}\right)$ & 678 & 52 & 2,4 & 2,5 & 0 & 14,7 & 0 & 1,6 & 3,5 \\
\hline $\mathrm{T}\left({ }^{0} \mathrm{C}\right)$ & 685 & 45 & 23,7 & 2,4 & 17 & 31,1 & 21,9 & 23,8 & 25,5 \\
\hline UR (\%) & 684 & 46 & 72,3 & 10,8 & 38,5 & 99,2 & 65,2 & 72,2 & 79,7 \\
\hline
\end{tabular}

a. Número de dias sem informação. b. Desvio Padrão. c. Percentil 25. d. Percentil 50. e. Percentil 75.

qualidade do ar. Para todas as cidades e poluentes analisados, os efeitos de maior magnitude foram observados quando se considerou a defasagem acumulada dos poluentes de zero a cinco dias. Pode-se observar que o $\mathrm{PM}_{10}$ apresentou a relação mais frequente com os eventos mórbidos estudados, particularmente as doenças respiratórias, apesar de apresentar níveis dentro dos padrões recomendados pela legislação brasileira, o que é consistente com as recomendações da Organização Mundial de Saúde da inexistência de limite seguro de efeito ${ }^{1}$. O PM $\mathrm{PM}_{10}$ esteve associado às internações por doenças respiratórias totais e em menores de cinco anos em todas as cidades investigadas. Relação entre os níveis de $\mathrm{PM}_{10}$ e internações por doenças cardiovasculares foi observada apenas em Belo Horizonte. Os efeitos do $\mathrm{PM}_{10}$ sobre o aparelho respiratório e cardiovascular são bem documentados, podendo estar relacionados ao surgimento, desencadeamento e piora de sintomas particularmente em populações vulneráveis como crianças e idosos ${ }^{11,12}$. PM é uma complexa mistura aérea de partículas sólidas, líquidas ou em fase mista que contém compostos orgânicos, inorgânicos e organometálicos em suspensão, com composição variando no tempo e no espaço. Em termos de diâmetro aerodinâmico o PM se classifica de acordo com seu tamanho. O PM ${ }_{10}$ são partículas grosseiras com menos de 10um de diâmetro; $\mathrm{PM}_{2,5}$ são partículas com diâmetro inferior a 2,5um e $\mathrm{PM}_{0,1}$ são partículas ultrafinas que incluem nanopartículas. Partículas 
Tabela 2. Risco Relativo percentual ${ }^{(a)}$ e Intervalo de Confiança de $95 \%$ para internações por doenças respiratórias totais, doenças respiratórias em menores de 5 anos e doenças cardiovasculares em maiores de 39 anos. Belo Horizonte, Minas Gerais, Brasil - julho/2004 a dezembro/2006.

\begin{tabular}{|c|c|c|c|c|c|c|c|c|c|c|}
\hline \multirow{3}{*}{$\begin{array}{l}\text { Poluente } \\
\text { PM10 }\end{array}$} & \multirow{3}{*}{$\begin{array}{l}\text { Defasagem } \\
\text { Dia corrente }\end{array}$} & \multicolumn{3}{|c|}{ DRT Totais $^{(\mathbf{b})}$} & \multicolumn{3}{|c|}{ DRC $<5 \operatorname{anos}^{(c)}$} & \multicolumn{3}{|c|}{ DCV > $39 \operatorname{anos}^{(d)}$} \\
\hline & & \multirow{2}{*}{$\begin{array}{r}\mathbf{R R} \% \\
-0,15\end{array}$} & \multicolumn{2}{|c|}{ IC (95\%) } & \multirow{2}{*}{$\begin{array}{r}\mathbf{R R} \% \\
-0,20\end{array}$} & \multicolumn{2}{|c|}{ IC (95\%) } & \multirow{2}{*}{$\begin{array}{r}\mathbf{R R} \% \\
2,32\end{array}$} & \multicolumn{2}{|c|}{ IC (95\%) } \\
\hline & & & $-1,19$ & 0,89 & & $-1,78$ & 1,41 & & 0,03 & 4,66 \\
\hline & Defasagem de 1 dia & 0,37 & $-0,27$ & 1,02 & 0,27 & $-0,71$ & 1,27 & 1,28 & $-0,04$ & 2,61 \\
\hline & Defasagem de 2 dias & 0,60 & 0,01 & 1,20 & 0,51 & $-0,40$ & 1,43 & 0,44 & $-0,74$ & 1,64 \\
\hline & Defasagem de 3 dias & 0,54 & $-0,01$ & 1,09 & 0,52 & $-0,33$ & 1,38 & $-0,20$ & $-1,33$ & 0,94 \\
\hline & Defasagem de 4 dias & 0,18 & $-0,33$ & 0,69 & 0,29 & $-0,49$ & 1,09 & $-0,64$ & $-1,73$ & 0,46 \\
\hline & Defasagem de 5 dias & $-0,47$ & $-1,34$ & 0,41 & $-0,16$ & $-1,50$ & 1,20 & $-0,89$ & $-2,77$ & 1,02 \\
\hline & Acumulado 5 dias & 1,06 & 0,41 & 1,72 & 1,25 & 0,25 & 2,26 & 2,29 & 0,96 & 3,64 \\
\hline \multirow[t]{7}{*}{$\mathrm{CO}$} & Dia corrente & 1,62 & $-2,57$ & 5,99 & 3,59 & $-2,91$ & 10,54 & 4,89 & $-4,24$ & 14,89 \\
\hline & Defasagem de 1 dia & 2,56 & $-0,10$ & 5,29 & 2,97 & $-1,14$ & 7,27 & 4,85 & $-0,69$ & 10,69 \\
\hline & Defasagem de 2 dias & 2,70 & 0,06 & 5,41 & 2,12 & $-1,91$ & 6,32 & 3,56 & $-1,71$ & 9,11 \\
\hline & Defasagem de 3 dias & 2,03 & $-0,49$ & 4,63 & 1,04 & $-2,81$ & 5,04 & 1,07 & $-3,92$ & 6,32 \\
\hline & Defasagem de 4 dias & 0,58 & $-1,70$ & 2,90 & $-0,26$ & $-3,75$ & 3,36 & $-2,53$ & $-7,10$ & 2,26 \\
\hline & Defasagem de 5 dias & $-1,63$ & $-5,26$ & 2,13 & $-1,77$ & $-7,33$ & 4,13 & $-7,13$ & $-14,42$ & 0,78 \\
\hline & Acumulado 5 dias & 8,05 & 5,25 & 10,93 & 7,85 & 3,54 & 12,35 & 4,19 & $-1,31$ & 10,00 \\
\hline \multirow[t]{7}{*}{$\mathrm{SO} 2$} & Dia corrente & 0,86 & $-0,70$ & 2,45 & 1,13 & $-1,29$ & 3,62 & 0,36 & $-2,98$ & 3,83 \\
\hline & Defasagem de 1 dia & 1,08 & 0,04 & 2,12 & 1,11 & $-0,49$ & 2,73 & 1,88 & $-0,18$ & 3,98 \\
\hline & Defasagem de 2 dias & 0,99 & $-0,12$ & 2,13 & 0,82 & $-0,89$ & 2,56 & 2,44 & 0,28 & 4,65 \\
\hline & Defasagem de 3 dias & 0,63 & $-0,49$ & 1,76 & 0,26 & $-1,43$ & 1,99 & 2,04 & $-0,09$ & 4,22 \\
\hline & Defasagem de 4 dias & $-0,03$ & $-1,05$ & 1,01 & $-0,54$ & $-2,11$ & 1,05 & 0,69 & $-1,28$ & 2,71 \\
\hline & Defasagem de 5 dias & $-0,97$ & $-2,52$ & 0,60 & $-1,60$ & $-3,99$ & 0,85 & $-1,57$ & $-4,98$ & 1,74 \\
\hline & Acumulado 5 dias & 2,58 & 1,53 & 3,64 & 1,16 & $-0,44$ & 2,78 & 5,93 & 3,79 & 8,12 \\
\hline
\end{tabular}

a. expressa a variação percentual no número médio diário de internações hospitalares para uma variação de $10 \mu \mathrm{g} / \mathrm{m} 3 \mathrm{na}$ concentração do poluente. b. internações por doenças respiratórias totais. c. internações por doenças respiratórias em menores de 5 anos. d. internações por doenças cardiovasculares em maiores de 39 anos.

Tabela 3. Risco Relativo percentual ${ }^{(a)}$ e Intervalo de Confiança de 95\% para internações por doenças respiratórias totais, doenças respiratórias em menores de 5 anos e doenças cardiovasculares em maiores de 39 anos. Betim, Minas Gerais, Brasil - janeiro/2002 a dezembro/2006.

\begin{tabular}{|c|c|c|c|c|c|c|c|c|c|c|}
\hline \multirow{3}{*}{$\begin{array}{l}\text { Poluente } \\
\text { PM10 }\end{array}$} & \multirow{3}{*}{$\begin{array}{l}\text { Defasagem } \\
\text { Dia corrente }\end{array}$} & \multicolumn{3}{|c|}{ DRT Totais ${ }^{(\mathbf{b})}$} & \multicolumn{3}{|c|}{$\mathrm{DRC}<5 \operatorname{anos}^{(\mathrm{c})}$} & \multicolumn{3}{|c|}{ DCV $>39$ anos $^{(d)}$} \\
\hline & & \multirow{2}{*}{$\begin{array}{l}\mathbf{R R} \% \\
-0,08\end{array}$} & \multicolumn{2}{|c|}{ IC (95\%) } & \multirow{2}{*}{$\begin{array}{c}\mathbf{R R} \% \\
0,45\end{array}$} & \multicolumn{2}{|c|}{ IC (95\%) } & \multirow{2}{*}{$\begin{array}{l}\mathbf{R R} \% \\
-0,13\end{array}$} & \multicolumn{2}{|c|}{ IC (95\%) } \\
\hline & & & $-1,56$ & 1,42 & & $-1,59$ & 2,54 & & $-2,08$ & 1,86 \\
\hline & Defasagem de 1 dia & 0,10 & $-0,74$ & 0,94 & 0,13 & $-1,01$ & 1,30 & 0,31 & $-0,73$ & 1,37 \\
\hline & Defasagem de 2 dias & 0,23 & $-0,52$ & 0,99 & 0,04 & $-0,98$ & 1,06 & 0,48 & $-0,48$ & 1,45 \\
\hline & Defasagem de 3 dias & 0,32 & $-0,40$ & 1,06 & 0,16 & $-0,82$ & 1,15 & 0,36 & $-0,60$ & 1,34 \\
\hline & Defasagem de 4 dias & 0,37 & $-0,35$ & 1,10 & 0,50 & $-0,50$ & 1,51 & $-0,03$ & $-1,01$ & 0,95 \\
\hline & Defasagem de 5 dias & 0,37 & $-0,94$ & 1,71 & 1,07 & $-0,76$ & 2,92 & $-0,71$ & $-2,48$ & 1,09 \\
\hline & Acumulado 5 dias & 1,33 & 0,48 & 2,18 & 2,38 & 1,20 & 3,56 & 0,27 & $-0,77$ & 1,32 \\
\hline
\end{tabular}

a. expressa a variação percentual no número médio diário de internações hospitalares para uma variação de $10 \mu \mathrm{g} / \mathrm{m} 3 \mathrm{na}$ concentração do poluente. b. internações por doenças respiratórias totais. c. internações por doenças respiratórias em menores de 5 anos. d. internações por doenças cardiovasculares em maiores de 39 anos.

menores têm maior capacidade de ultrapassar as barreiras fisiológicas e alcançar as diversas estruturas do corpo além dos pulmões, induzindo diversos efeitos através de múltiplos mecanismos, incluindo lesão endotelial, aumento da resposta inflamatória, estresse oxidativo, disfunção autonômica e dano na mitocôndria além de efeitos genotóxicos ${ }^{13,14}$. 
Tabela 4. Risco Relativo percentual ${ }^{(\mathrm{a})}$ e Intervalo de Confiança de $95 \%$ para internações por doenças respiratórias totais, doenças respiratórias em menores de 5 anos e doenças cardiovasculares em maiores de 39 anos. Contagem, Minas Gerais, Brasil - dezembro/2004 a novembro/2006.

\begin{tabular}{|c|c|c|c|c|c|c|c|c|c|c|}
\hline \multirow{3}{*}{$\begin{array}{l}\text { Poluente } \\
\text { PM10 }\end{array}$} & \multirow{3}{*}{$\begin{array}{l}\text { Defasagem } \\
\text { Dia corrente }\end{array}$} & \multicolumn{3}{|c|}{ DRT Totais ${ }^{(\mathbf{b})}$} & \multicolumn{3}{|c|}{ DRC $<5 \operatorname{anos}^{(c)}$} & \multicolumn{3}{|c|}{ DCV > $39 \operatorname{anos}^{(d)}$} \\
\hline & & \multirow{2}{*}{$\begin{array}{c}\text { RR\% } \\
1,41\end{array}$} & \multicolumn{2}{|c|}{ IC (95\%) } & \multirow{2}{*}{$\begin{array}{c}\text { RR\% } \\
1,13\end{array}$} & \multicolumn{2}{|c|}{ IC $(95 \%)$} & \multirow{2}{*}{$\begin{array}{l}\text { RR\% } \\
-0,77\end{array}$} & \multicolumn{2}{|c|}{ IC (95\%) } \\
\hline & & & $-0,15$ & 2,99 & & $-1,12$ & 3,43 & & $-2,58$ & 1,07 \\
\hline & Defasagem de 1 dia & 0,61 & $-0,29$ & 1,53 & 0,55 & $-0,78$ & 1,89 & $-0,53$ & $-1,57$ & 0,52 \\
\hline & Defasagem de 2 dias & 0,06 & $-0,82$ & 0,94 & 0,14 & $-1,11$ & 1,42 & $-0,25$ & $-1,21$ & 0,71 \\
\hline & Defasagem de 3 dias & $-0,26$ & $-1,12$ & 0,60 & $-0,08$ & $-1,29$ & 1,14 & 0,07 & $-0,86$ & 0,99 \\
\hline & Defasagem de 4 dias & $-0,36$ & $-1,15$ & 0,44 & $-0,13$ & $-1,26$ & 1,01 & 0,42 & $-0,45$ & 1,30 \\
\hline & Defasagem de 5 dias & $-0,22$ & $-1,55$ & 1,13 & $-0,01$ & $-1,92$ & 1,94 & 0,82 & $-0,69$ & 2,35 \\
\hline & Acumulado 5 dias & 1,23 & 0,32 & 2,15 & 1,61 & 0,26 & 2,96 & $-0,25$ & $-1,30$ & 0,80 \\
\hline \multirow[t]{7}{*}{$\mathrm{SO} 2$} & Dia corrente & 0,53 & $-0,84$ & 1,93 & $-0,08$ & $-2,03$ & 1,90 & $-0,29$ & $-1,90$ & 1,35 \\
\hline & Defasagem de 1 dia & 0,69 & $-0,05$ & 1,43 & 0,32 & $-0,77$ & 1,42 & $-0,17$ & $-1,03$ & 0,69 \\
\hline & Defasagem de 2 dias & 0,69 & $-0,09$ & 1,48 & 0,54 & $-0,61$ & 1,71 & $-0,07$ & $-0,96$ & 0,83 \\
\hline & Defasagem de 3 dias & 0,54 & $-2,26$ & 1,35 & 0,58 & $-0,57$ & 1,75 & 0,00 & $-0,89$ & 0,91 \\
\hline & Defasagem de 4 dias & 0,24 & $-0,50$ & 0,98 & 0,45 & $-0,59$ & 1,51 & 0,07 & $-0,77$ & 0,88 \\
\hline & Defasagem de 5 dias & $-0,22$ & $-1,49$ & 1,07 & 0,14 & $-1,68$ & 1,99 & 0,09 & $-1,42$ & 1,62 \\
\hline & Acumulado 5 dias & 2,49 & 1,74 & 3,25 & 1,96 & 0,86 & 3,08 & $-0,39$ & $-1,24$ & 0,47 \\
\hline
\end{tabular}

a. expressa a variação percentual no número médio diário de internações hospitalares para uma variação de $10 \mu \mathrm{g} / \mathrm{m} 3 \mathrm{na}$ concentração do poluente. b. internações por doenças respiratórias totais. c. internações por doenças respiratórias em menores de 5 anos. d. internações por doenças cardiovasculares em maiores de 39 anos.

$\mathrm{O} \mathrm{SO}_{2}$, avaliado em Belo Horizonte e Contagem apresentou relação estatisticamente significativa com internações por DRT nos dois municípios e com DRC em Contagem. A relação entre $\mathrm{o} \mathrm{SO}_{2} \mathrm{e}$ as internações por doenças respiratórias são evidência dos efeitos irritativos desse poluente $^{11}$. Internações por $\mathrm{DCV}$ se relacionaram ao $\mathrm{SO}_{2}$ apenas em Belo Horizonte. Os achados do presente estudo são consistentes com a associação encontrada entre o $\mathrm{PM}_{10}, \mathrm{PM}_{2,5}, \mathrm{CO}, \mathrm{NO}_{2}, \mathrm{SO}_{2} \mathrm{em}$ revisão e meta-análise de 34 publicações onde se avalia o infarto do miocárdio ${ }^{15}$. Na RMBH, o CO foi aferido apenas na capital e esteve relacionado às internações por DRT e DRC. Embora não tenha sido verificada relação entre todos os poluentes avaliados no presente estudo com as internações por doenças cardiovasculares, sabe-se que estas são uma das principais causas de internação e demandam alto custo para o sistema de saúde. Estima-se, por exemplo, que a redução dos níveis de $\mathrm{PM}_{2.5}$ de $3.9 \mu \mathrm{g} / \mathrm{m}^{3}$ representaria uma economia de três milhões de dólares anuais para os cofres públicos nos Estados Unidos ${ }^{16}$.

Obteve-se um bom ajuste dos modelos apesar dos pequenos números de internações em Betim e Contagem, no entanto, deve-se ressaltar que os achados representam as internações registradas nas AIH que se referem aos pacientes atendidos pelo SUS. O registro de internações provenientes dos hospitais não conveniados ao SUS estão disponíveis a partir de 2008, onde correspondem a $20 \%$ do total de internações em Belo Horizonte, $17 \%$ e $11 \%$ em Betim e Contagem, respectivamente. A ausência de dados dos hospitais não conveniados ao SUS, correspondentes ao período de estudo, não nos permitiu analisar as internações como um todo, o que pode ter limitado a interpretação dos achados enquanto representativos de toda a população.

A representatividade dos parâmetros aferidos pelas estações de monitoramento é uma outra questão a ser considerada. De acordo com o relatório do órgão ambiental de Minas Gerais ${ }^{7}$, devido à localização e número de estações de monitoramento, o $\mathrm{PM}_{10}$ é o poluente que reflete mais precisamente a exposição da população, portanto, para os demais resultados, os dados devem ser vistos com cautela.

Belo Horizonte tem característica mista de fontes de poluição (indústria e veículos), enquanto que em Contagem e Betim predominam fontes de poluentes provenientes de indústrias. Dentre as cidades brasileiras estudadas, a cidade de Vitória (ES), também com característica mista de fontes de poluição, foi avaliada com a mesma metodologia do presente trabalho e apresentou risco relativo de internações por DRT e DRC associado aos níveis de $\mathrm{PM}_{10}$ de maior magnitude ${ }^{17}$. Vale ressaltar que os níveis médios anuais de $\mathrm{PM}_{10}$ em Vitória foram mais elevados que em Belo Ho- 
rizonte no período de estudo. Em Cubatão, onde predominam fontes provenientes de indústrias, $\mathrm{o}$ risco de internações por doenças respiratórias foi também de maior magnitude ${ }^{18}$ que em Betim e Contagem. Nessas cidades, os níveis de $\mathrm{PM}_{10}$ foram similares (Betim) ou maiores (Contagem).

Como já apontado por Laden et al. ${ }^{19}$, a composição do $\mathrm{PM}_{10}$ é dependente das fontes de emissão e sua toxicidade está relacionada aos seus componentes, que diferem de acordo com a localidade e origem ${ }^{20}$. A comparação apenas de localidades e efeitos de acordo com a predominância da fonte emissora, poderia ser testada, como uma primeira abordagem, comparando cidades com características de emissão similares em metanálise.

\section{Colaboradores}

N Gouveia: contribuição na concepção, análise e interpretação dos resultados, elaboração do rascunho e aprovação do manuscrito final. AP Leon: contribuição na concepção, análise e interpretação dos resultados, revisão e aprovação do manuscrito final. W Junger e JF Lins: contribuição na concepção, análise e interpretação dos resultados, revisão crítica e aprovação final do manuscrito. CU Freitas: contribuição no planejamento, análise e interpretação dos resultados, revisão crítica e aprovação do manuscrito final.
Estudos com metodologia similar ao presente trabalho vêm sendo realizados nas cidades brasileiras que contam com monitoramento da qualidade do ar, com a finalidade principal de contribuir para a discussão de medidas mitigadoras da poluição atmosférica, por parte da população e dos tomadores de decisão. Estas iniciativas de ar limpo para serem eficazes requerem a discussão dos diversos setores relacionados com o transporte, a indústria e o uso do solo em conjunto com os usuários ${ }^{21}$. A tomada de decisão em um fórum democrático, levando em consideração tanto ganhos diretos em saúde quanto em qualidade de vida só contribuirá para a saúde e bem - estar da população.

\section{Agradecimentos}

Agradeço ao Ministério da Saúde pelo financiamento deste trabalho como parte do "Projeto de avaliação do impacto na saúde dos níveis de poluição atmosférica nas cidades brasileiras e das políticas de controle da poluição do ar por veículos automotores", aprovado pelo comitê de Ética em Pesquisa da Faculdade de Medicina da USP. 


\section{Referências}

1. World Health Organization (WHO).WHO air quality guidelines global update 2005: Report on a working group meeting. 2005 Oct 18-20, Bonn, Germany.

2. Hoek G, Krishnan RM, Beelen R, Peters A, Ostro B, Brunekreef B, Kaufman JD. Long-term air pollution exposure and cardio-respiratory mortality: a review. Environ Health 2013; 12(1):43.

3. Atkinson RW, Kang S, Anderson HR, Mills IC, Walton HA. Epidemiological time series studies of PM2.5 and daily mortality and hospital admissions: a systematic review and meta-analysis. Thorax 2014; 69(7):660665.

4. Song Q, Christiani DC, Wang X, Ren J. The global contribution of outdoor air pollution to the incidence, prevalence, mortality and hospital admission for chronic obstructive pulmonary disease: a systematic review and meta-analysis. Int J Environ Res Public Health 2014; 11(11):11822-11832.

5. Brook RD, Rajagopalan S, Pope CA, Brook JR, Bhatnagar A, Diez-Roux AV, Holguin F, Hong Y, Luepker RV, Mittleman MA, Peters A, Siscovick D, Smith Junior SC, Whitsel L, Kaufman JD, American Heart Association Council on Epidemiology and Prevention, Council on the Kidney in Cardiovascular Disease, Council on Nutrition, Physical Activity and Metabolism. Particulate matter air pollution and cardiovascular disease: an update to the scientific statement from the American Heart Association. Circulation 2010; 121(21):2331-2378.

6. Romieu I, Gouveia N, Cifuentes LA, Leon AP, Junger W, Vera J, Strappa V, Hurtado-Díaz M, Miranda-Soberanis V, Rojas-Bracho L, Carbajal-Arroyo L, Tzintzun-Cervantes G, HEI Health Review Committee. Multicity study of air pollution and mortality in Latin America (the ESCALA study). Res Rep Health EffInst 2012; (171):5-86.

7. Fundação Estadual do Meio Ambiente (FEAM). Monitoramento da Qualidade do Ar na Região Metropolitana de Belo Horizonte no Ano de 2012 - Relatório Técnico [Internet]. $2015 \mathrm{Nov}$ [acessado $2016 \mathrm{Abr}$ 2]:[cerca de 52p]. Disponível em: http://www.feam. br/images/stories/2016/QUALIDADE_AR/Monitoramento_da_Qualidade_do_Ar_na_RMBH_de_2012. pdf.

8. Instituto Brasileiro de Geografia e Estatística (IBGE) [Site Internet]. [acessado 2016 Abr 2]. Disponível em: http://www.cidades.ibge.gov.br/xtras/uf.php?lan$\mathrm{g}=\&$ coduf $=31$ \&search $=$ minas-gerais

9. Junger WL. Análise, imputação de dados e interfaces computacionais em estudos de séries temporais epidemiológicas [tese]. Rio de Janeiro: Universidade Estadual do Rio de Janeiro; 2008.

10. Schwartz J. The distributed lag between air pollution and daily deaths. Epidemiology 2000; 11(3):320-326.

11. Ghorani-Azam A, Riahi-Zanjani B, Balali-Mood M. Effects of air pollution on human health and practical measures for prevention in Iran. J Res Med Sci 2016; 21:65.
12. Mehta S, Shin H, Burnett R, North T, Cohen AJ. Ambient particulate air pollution and acute lower respiratory infections: a systematic review and implications for estimating the global burden of disease. Air Qual Atmos Health 2013; 6(1):69-83.

13. Traboulsi H, Guerrina N, Iu M, Maysinger D, Baglole CJ. Inhaled Pollutants: The Molecular Scene behind Respiratory and Systemic Diseases Associated with Ultrafine Particulate Matter. Int J Mol Sci 2017; 18(2):243.

14. Meng X, Zhang Y, Yang KQ, Yang YK, Zhou XL. Potential Harmful Effects of PM2.5 on Occurrence and Progression of Acute Coronary Syndrome: Epidemiology, Mechanisms, and Prevention Measures. Int $J$ Environ Res Public Health 2016; 13(8):748.

15. Mustafic H, Jabre P, Caussin C, Murad MH, Escolano S, Tafflet M, Périer MC, Marijon E, Vernerey D, Empana JP, Jouven X. Main air pollutants and myocardial infarction: a systematic review and meta-analysis. JAMA 2012; 307(7):713-721.

16. Shah AS, Langrish JP, Nair H, McAllister DA, Hunter AL, Donaldson K, Newby DE, Mills NL. Global association of air pollution and heart failure: a systematic review and meta-analysis. Lancet 2013; 382(9897):1039-1048.

17. Freitas CU, Leon AP, Junger W, Gouveia N. Poluição do ar e impactos na saúde em Vitória, Espírito Santo. Rev Saude Publica 2016; 50(4):1-9.

18. Nardocci AC, Freitas CU, Leon AP, Junger W, Gouveia N. Poluição do ar e doenças respiratórias e cardiovasculares: estudo de séries temporais em Cubatão, São Paulo, Brasil. Cad Saude Publica 2013; 29(9):18671876.

19. Laden F, Neas LM, Dockery DW, Schwartz J. Association of fine particulate matter from different sources with daily mortality in six U.S. cities. EHP 2000; 108(10):941-947.

20. Bell ML, Dominici F, Ebisu K, Zeger SC, Samet JM. Spatial and temporal variation in PM2.5 chemical composition in the United States for health effects studies. EHP 2007; 115(7):989-995.

21. Nieuwenhuijsen MJ. Urban and transport planning, environmental exposures and health-new concepts, methods and tools to improve health in cities. Environ Health 2016; 15(38 Supl. 1):161-171.

Artigo apresentado em 23/11/2017

Aprovado em 12/03/2018

Versão final apresentada em 15/03/2018 
